\title{
WASH Benefits Bangladesh trial: system for monitoring coverage and quality in an efficacy trial
}

\author{
Mahbubur Rahman ${ }^{1 *}$ D, Sania Ashraf', Leanne Unicomb', A. K. M. Mainuddin', Sarker Masud Parvez', \\ Farzana Begum', Kishor Kumar Das', Abu Mohd. Naser ${ }^{3}$, Faruqe Hussain ${ }^{1}$, Thomas Clasen ${ }^{3}$, Stephen P. Luby ${ }^{4}$, \\ Elli Leontsini ${ }^{2}$ and Peter J. Winch ${ }^{2}$
}

\begin{abstract}
Background: Researchers typically report more on the impact of public health interventions and less on the degree to which interventions were followed implementation fidelity. We developed and measured fidelity indicators for the WASH Benefits Bangladesh study, a large-scale efficacy trial, in order to identify gaps between intended and actual implementation.

Methods: Community health workers (CHWs) delivered individual and combined water, sanitation, handwashing (WSH) and child nutrition interventions to 4169 enrolled households in geographically matched clusters. Households received free enabling technologies (insulated water storage container; sani-scoop, potty, double-pit, pour-flush latrine; handwashing station, soapy-water storage bottle), and supplies (chlorine tablets, lipid-based nutrient supplements, laundry detergent sachets) integrated with parallel behavior-change promotion. Behavioral objectives were drinking treated, safely stored water, safe feces disposal, handwashing with soap at key times, and age-appropriate nutrition behaviors. We administered monthly surveys and spot-checks to households from randomly selected clusters for 6 months early in the trial. If any fidelity measures fell below set benchmarks, a rapid response mechanism was triggered.
\end{abstract}

Results: In the first 3 months, functional water seals were detected in 33\% (14/42) of latrines in the sanitation only arm; 35\% (14/40) for the combined WSH arm; and 60\% (34/57) for the combined WSH and Nutrition arm, all falling below the pre-set benchmark of $80 \%$. Other fidelity indicators met the 65 to $80 \%$ uptake benchmarks. Rapid qualitative investigations determined that households concurrently used their own latrines with broken water seals in parallel with those provided by the trial. In consultation with the households, we closed pre-existing latrines without water seals, increased the CHWs' visit frequency to encourage correct maintenance of latrines with water seals, and discouraged water-seal removal or breakage. At the sixth assessment, 86\% (51/59) of households were in sanitation only; $92 \%$ (72/78) in the combined WSH; and 93\% (71/76) in the combined WSH and Nutrition arms had latrines with functional water seals.

Conclusions: An intensive implementation fidelity monitoring and rapid response system proved beneficial for this efficacy trial. To implement a routine program at scale requires further research into an adaptation of fidelity monitoring that supports program effectiveness.

(Continued on next page)

\footnotetext{
* Correspondence: mahbubr@icddrb.org

${ }^{1}$ Environmental Intervention Unit, Enteric and Respiratory Disease Program,

Infectious Disease Division, International Centre for Diarrheal Disease

Research, Bangladesh (icddr,b), Dhaka, Bangladesh

Full list of author information is available at the end of the article
}

(c) The Author(s). 2018 Open Access This article is distributed under the terms of the Creative Commons Attribution 4.0 International License (http://creativecommons.org/licenses/by/4.0/), which permits unrestricted use, distribution, and reproduction in any medium, provided you give appropriate credit to the original author(s) and the source, provide a link to the Creative Commons license, and indicate if changes were made. The Creative Commons Public Domain Dedication waiver (http://creativecommons.org/publicdomain/zero/1.0/) applies to the data made available in this article, unless otherwise stated. 
(Continued from previous page)

Trial registration: WASH Benefits Bangladesh: ClinicalTrials.gov, ID: NCT01590095. Registered on 30 April 2012.

Keywords: WASH Benefits, Implementation fidelity, Water quality, Sanitation, Handwashing, Child nutrition, Efficacy, Behavior change, Cluster randomized controlled trial, Bangladesh

\section{Background}

Researchers typically report on the health impact and/or behavior change of large-scale, often complex public health interventions that include methods to persuade target groups to adopt an innovation such as a health-related technology or behavior [1]. Intervention impact may vary depending on programmatic efficiency, and political and social contexts. Contextual factors in both implementation and operation may moderate the impact of a planned intervention [2]. Very few community trials have reported on implementation fidelity, i.e., the degree to which an intervention is delivered as intended [3-5].

Implementation fidelity is an important fundamental tool for assessing the implementation process [6-8] which can help to explain the association between intervention and outcomes [4]. When limited or no impact is detected in an intervention trial, it is difficult to determine whether this occurred because the intervention was ineffective, or intervention uptake and implementation quality was poor [9]. Fidelity measurement can additionally enhance the credibility of intervention impact and transferability to other settings [10]. Conceptually, implementation fidelity has been defined using five elements: adherence to the planned intervention, exposure or dose, quality of delivery, participant responsiveness and program differentiation $[4,5]$. Of these, quality of delivery can be measured against benchmarks set by the intervention implementers, to relate to adequate participant exposure $[4,11]$. Determining whether fidelity indicators meet benchmarks early in the implementation period can guide corrective action through investigations of shortfalls in intervention delivery or uptake.

Among components of water, sanitation, and hygiene (WASH) interventions, there are some that can be initiated at one time point; e.g., water treatment or handwashing. However, sanitation interventions that include latrine construction require a series of steps to be completed; for example, negotiating location with the householders, digging the pit, producing the cement rings and slab, installing rings and slabs, and installing the above-ground (super) structure. Not all steps are necessarily completed as indicated; latrines may be half-constructed, or constructed and never used, or modified post construction. These conditions can limit the ability of latrines to separate feces from the environment, resulting in low implementation fidelity. WASH Benefits was a large-scale, cluster-randomized efficacy trial that aimed to assess the impact of improvements in water quality, sanitation, handwashing, and child nutrition and feeding - alone and in combination, on child health [12]. Therefore, it was necessary to achieve high levels of uptake of both enabling technologies (latrines, handwashing stations, etc.) and the related behaviors. To this end, we put in place an intensive system for monitoring intervention delivery, described in detail elsewhere [13]. Here, we describe development and use of fidelity measure indicators for the WASH Benefits Bangladesh study, as part of a three-paper series on WASH Benefits Intervention Delivery and Performance $[13,14]$. We illustrate the benefits of early review of a rigorous, real-time fidelity assessment for this trial that required complex hardware delivery and installation, as well as parallel behavior-change promotion. We describe the use of benchmarks to signal early implementation problems, particularly for latrine installation that required qualitative investigation and course correction. Monitoring fidelity indicators throughout the intervention period is described in the third paper of this series [14].

\section{Methods}

\section{WASH Benefits intervention design}

The WASH Benefits Bangladesh randomized controlled trial as conducted across 720 clusters comprising six to eight geographically proximate households identified to have a pregnant mother at enrollment, totaling 4169 intervention households in four districts. Details of the study design are described elsewhere [12]. In summary, there were six intervention arms that included free provision of enabling technologies and supplies depicted in Table 1, integrated with parallel behavior-change promotion. The arms were (1) drinking-water treatment and safe storage, (2) sanitation, (3) handwashing, (4) child nutrition, (5) water, sanitation, handwashing (WSH), and (6) nutrition plus WSH. Locally recruited community health workers (CHWs), residents of the study villages, were trained to deliver and promote the interventions during regular home visits. Each $\mathrm{CHW}$ was responsible for one cluster of one intervention arm. Six intervention and two control geographically matched clusters were grouped into a trial block.

Among households in the individual and combined sanitation arms, a sanitation team (comprising a research scientist, sanitation engineers from the implementing agency, village education resource center (VERC) and field attendants) installed study-provided 
Table 1 Description of WASH Benefits trial interventions, fidelity measures and benchmarks, Bangladesh, 2012-2015

\begin{tabular}{|c|c|c|}
\hline Interventions $^{a}$ & Provided to eligible households ${ }^{b}$ & Fidelity measures ${ }^{\complement}$ (benchmark for uptake ${ }^{c}$ ) \\
\hline Water quality & $\begin{array}{l}\text { Free supply of chlorine tablets (Aquatabs }{ }^{\oplus} \text { brand, } \\
\text { sodium dichloroisocyanurate) with a } 10-\mathrm{L} \text { insulated } \\
\text { water storage container (Fig. 1a) } \\
\text { Storage container, } N=2160 \\
\text { Tablets: } 64,800 / \text { month }\end{array}$ & $\begin{array}{l}\text { Presence of chlorine in stored water detected using } \\
\text { a HACH color-wheel colorimeter }(>0.2 \mathrm{mg} / \mathrm{L}) \\
(\mathrm{HACH} \text { LANGE GmbH, USA) }(65 \%)\end{array}$ \\
\hline Sanitation & $\begin{array}{l}\text { Double-pit improved latrine; sani- scoop, a locally } \\
\text { developed tool for child and animal feces handling } \\
\text { and disposal (Sultana et al.); potties (Faruqe) (Fig. 1b) } \\
\text { Latrine installed: } 4533 \\
\text { Child potty: } 4384 \\
\text { Sani-scoop: } 6303\end{array}$ & $\begin{array}{l}\text { Reported location and method of disposal for } \\
\text { child's last bowel motion (65\%) } \\
\text { Spot check for sani-scoop that easily accessible to } \\
\text { mother ( } 80 \%) \\
\text { Spot check for visible potty (easily accessible to } \\
\text { mother) ( } 80 \%) \\
\text { Spot check of designated household latrines; a } \\
\text { functional water seal (80\%) }\end{array}$ \\
\hline Handwashing & $\begin{array}{l}\text { A handwashing station comprised of a bucket with } \\
\text { a fitted tap ( } 40 \mathrm{~L} \text { near the latrine and } 16 \mathrm{~L} \text { near the } \\
\text { cooking area), a soapy-water bottle (Hulland et al. } \\
\text { [18]) and free bi-weekly supply of detergent for } \\
\text { soapy-water preparation (Fig. 1c) } \\
\text { Station for latrine and kitchen: } 4320\end{array}$ & $\begin{array}{l}\text { Spot check for handwashing location near the toilet } \\
\text { and the cooking area, and for the presence of } \\
\text { water and soap ( } 65 \% \text { with at least one with soap } \\
\text { and water present) }\end{array}$ \\
\hline Child nutrition & $\begin{array}{l}\text { Promotion of exclusive breastfeeding up to } 6 \\
\text { months of age, complementary feeding and } \\
\text { supplementation using lipid-based nutrient } \\
\text { supplements (LNS; Nutriset, France) for children } \\
\text { aged 6-24 months (Fig. 1d). } \\
\text { Total sachets distributed: 1,579,900 }\end{array}$ & $\begin{array}{l}\text { Reported messages on infant/child nutrition and/or } \\
\text { lipid- based nutrient supplements (LNS) local name; } \\
\text { Sonamoni ( } 80 \%) \\
\text { Inspection of number of sachets of Sonamoni for } \\
\text { children }>6 \text { months of age, consistent with use of } \\
2 \text { sachets per day ( } 70 \%)\end{array}$ \\
\hline
\end{tabular}

${ }^{a}$ Combined arms: The interventions were delivered sequentially in the following order: sanitation, handwashing. Water treatment, with a minimum of 21 days between each start date. LNS was delivered when the child reaches 6 months of age; fidelity indicators and benchmarks as for individual components; ${ }^{b}$ eligible households were those directly enrolled by the study in the individual water, handwashing, and nutrition arms. For arms that received the sanitation intervention, interventions were provided to all households in the compound of the index household; potties were provided to all households in the compound of the index household who had children too young to use a latrine. 'based on unannounced visits by the Fidelity Assessment Team; does not include hardware replacements made during the course of the trial

latrines, independent of other intervention hardware delivery, with the first latrine installed in December, 2012 and the final in February, 2014. The latrine model, referred to as "double pit" (alternatively named dual pit, twin pit, two-pit, Fossa Alterna) is a traditional improved pit latrine that comprises two unconnected pits; one in use and one for which households transfer the above-ground (super) structure when the first pit is full [15]. This model uses a water seal (siphon), leading from the latrine to the pit that prevents odors from coming from the pit below into the toilet area (Fig. 2). The installation process involved multiple quality assurance steps. Latrine components were manufactured by pre-approved suppliers that met project-set standards of production; a subset of components was checked for compliance by breaking $5 \%$ of the concrete rings, slab and lid purchased for the project with a hammer. We checked the metal reinforcement of the same components by using metal detectors. If any evaluated component failed the quality check, the entire lot was rejected. The sanitation team used a checklist to verify complete latrine installation.

The enabling technologies for other intervention components included child potties [16], feces collecting sani-scoops [17], insulated drinking-water storage containers, handwashing stations, and soapy-water storage bottles [18]. Supplies provided by the project included laundry detergent sachets for soapy-water preparation, chlorine tablets (sodium dichloroisocyanurate, Aquatabs ${ }^{\ominus}$, Medentech, Wexford, Ireland), and lipid-based nutrient supplements (LNS, Nutriset, Malauanay, France) (Table 1, Fig. 1). Behavioral objectives were drinking treated, safely stored water, safe feces disposal, handwashing with soap at key times, and age-appropriate nutrition behaviors (pregnancy to 24 months). The interventions were rolled out from September 2012 to the first 10 geographically proximate trial blocks, monitored and refined during a 2-3 month period, then rolled out to the remaining trial blocks in nine phases to accommodate the logistics and the large number of staff members needed to deploy quality interventions [13].

\section{Development of fidelity criteria and critical benchmarks}

Fidelity measurements were designed to capture the quality of intervention delivery, which we measured as the presence of fully functional hardware, exposure to $\mathrm{CHW}$ visits and indicators of technology and supply use. A team comprising the principal and co-investigators, international collaborators, local scientific staff and implementation specialists developed a priori fidelity indicators that could be easily measured, and critical benchmarks that could be feasibly attained (Table 1). 
a
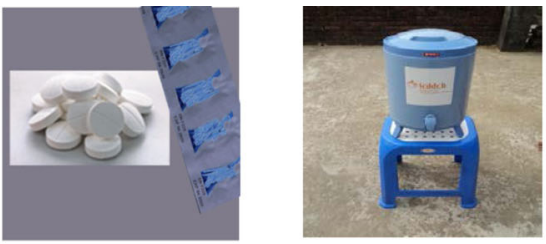

b
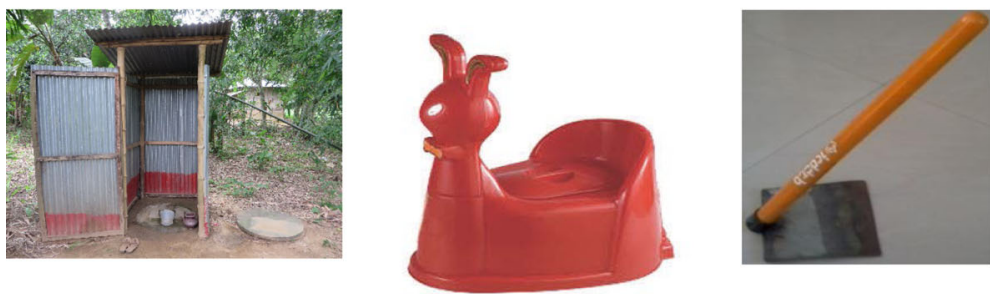

C

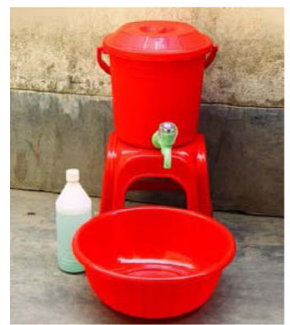

d

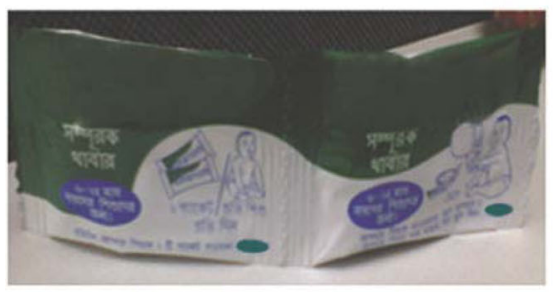

Fig. 1 WASH Benefits interventions products. a chlorine tablets and water storage container. b double-pit, pour-flush latrine, child potty and sani-scoop. $\mathbf{c}$ handwashing station. d lipid-based nutrient supplement (LNS)

These included observations of the presence, functionality, condition and signs of use of delivered hardware using a structured evaluation. For double-pit latrine functionality this included observation of an intact functional water seal (Fig. 2, Table 1). We conducted qualitative studies to develop specific definitions of 'easily accessible to mother', defined as producing technologies/ supplies within $10 \mathrm{~s}$ for child potties, sani-scoops, or the stock of LNS sachets, and checked for adequate delivery. These fidelity indicators and benchmarks were also used to monitor uptake throughout the trial, result of which are reported elsewhere [14].

\section{Data collection and analysis}

We administered surveys and spot checks during unannounced fidelity assessment visits every month, from November, 2012, 2 months after intervention delivery commenced, and for the next 6 months. We surveyed more intensely in the first 4 months, all intervention households from four randomly selected blocks per month, of the 10 initial trial blocks where the interventions had already been delivered $(n=8 \times 6 \times 4$ households). We surveyed four randomly selected households per intervention cluster from eight randomly selected blocks per month, of 20 trial blocks where the interventions had already been delivered $(n=4 \times 6 \times 8$ households), in the remaining months. The sample size for assessing intervention fidelity was calculated based on feasibility and convenience, no statistical method was applied. We randomly selected the households each month to ensure representativeness. Measures were made more intensely in the early period because WASH Benefits was a large complex intervention with inevitable deviations from optimal implementation. We aimed for early identification of problem to guide corrective measures to avoid deviation in the subsequent intervention blocks.

We conducted both surveys and spot checks; the survey provided a snapshot of the attitudes and behaviors including thoughts, meaningful opinions, and comments among the target population. Spot checks, conducted in real time, provided objective measures of hardware functionality and use indicating on the ground program operation, and any unexpected developments affecting the program and its objectives. 
a

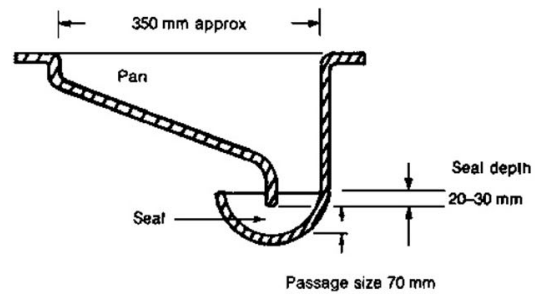

C

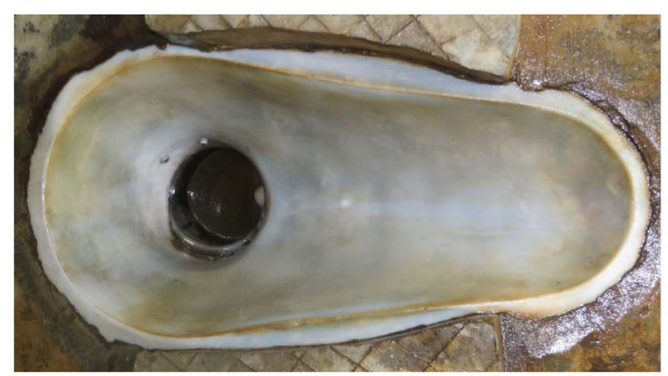

b

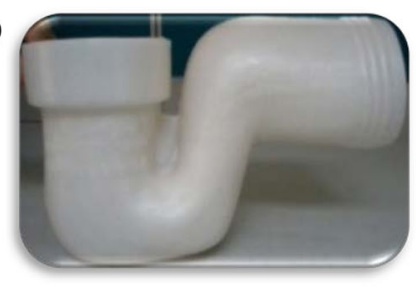

d

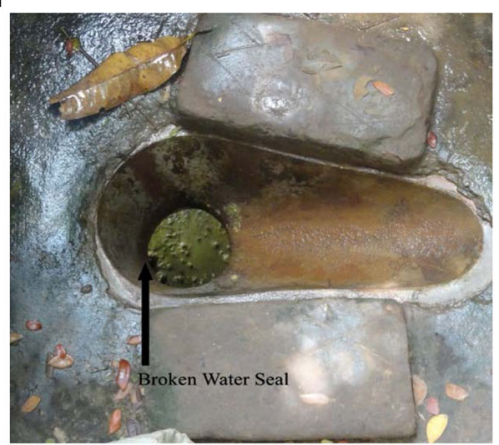

Fig. 2 Latrine water seal: schematic diagram and example of a broken water seal; a diagrammatic cross section of the water seal. b photo of water seal model in WASH Benefits latrines. c with intact water seal (water is visible). $\mathbf{d}$ with broken water seal recognized because of the ability to see feces

Field workers collected data on smartphones, using a survey and spot-check form on the presence (double-pit latrine with super structure, slab/pan, sani-scoop and potty for three or more children), functionality (double-pit latrine: superstructure is in good condition, slab/pan is in good condition, feces have not flowed out, functional water seal; potty and sani-scoop: not broken and easily accessible) condition and signs of use (double-pit latrine: wet floor and pan, water in water seal, feces in pan/water seal, pathway to latrine suggest regular use is clear, without grass or any barrier, water container present; potty: visible sign of feces inside/on the pot/removable pot, sani-scoop: visible sign of feces on the sani-scoop, not broken)of provided technologies, and on reported corresponding behaviors (use of double-pit latrine, use of potty and sani-scoop, disposal practices for child and animal feces) (Table 1). Data collectors checked for completeness and consistency of forms after each visit. Data were stored in a secure server in a SQL database, and checked for validity and data consistency. We calculated frequencies of fidelity measures for each intervention component (Table 1) using STATA 13 (StataCorp LP, College Station, TX, USA).

The data were rapidly assessed and circulated as standardized reports within 30 days of data collection. If any of the monthly fidelity measures were below critical benchmarks, then a rapid response mechanism was triggered. Investigators including anthropologists trained in qualitative investigation and not involved in project implementation reviewed monitoring and process documentation previously filed from routine monthly field staff and $\mathrm{CHW}$ meetings in the low-performing areas. They also visited sites, conducted informal discussions with CHWs, their supervisors, field staff, and study participants to identify the underlying problem(s). The findings were summarized and discussed to develop appropriate actions in response. These included additional classroom training and on the job training for CHWs, their supervisors, and field staff, on how to problem-solve to achieve the pre-set benchmark.

\section{Results}

Intervention fidelity achievements

By the first month, the water treatment fidelity indicator, residual chlorine in stored water, met the benchmark (65\%), as did the reported safe feces disposal indicator (critical benchmark 65\%), sani-scoop easily accessible to the mother $(80 \%)$ and reporting a CHW dissemination visit in the preceding month (90\%; data not shown). At least one handwashing station with soap and water present (65\%) was attained by the third month. Among households with children $>6$ months of age, the benchmark for observable stocks of lipid-based nutrient supplements (LNS) consistent with use of two sachets per day $(80 \%)$ was met when the child became eligible to consume it (fifth and sixth assessment), as was hearing messages on infant/child nutrition and LNS (70\%; data not shown). Reported child feces disposal (last child defecation event disposed into the latrine or a designated pit) met the targeted benchmark (65\%) at each fidelity 
assessment round. However, in three clusters, at the 1and 3-month assessments, following the distribution of potties to all children under the age of 3 years living in the target household's compound, this indicator was below the benchmark. Moreover, the sanitation benchmark of $80 \%$ of household latrines with a functional water seal was not met by the fourth assessment (Fig. 3a).

\section{Investigation of shortfall in sanitation implementation benchmarks: Latrine maintenance and use}

The reasons for which $<80 \%$ of household latrines were found with a functional water seal were explored from the third assessment month by staff responsible for latrine installation, the qualitative team, CHWs and their supervisors. The qualitative investigation found that several households intentionally removed or broke the water seals in the newly installed, trial provided latrines (Figs. 2 and $3 b$ ). This is because they perceived that the water seals required extra water to flush the fecal contents around the S-shaped bend into the pit below and might fill the pits rapidly, blocking easy flow of feces into the pit, or causing water to splash during defecation. The recipients did not pour an adequate volume of water into the toilet during post-defecation cleansing, and thus failed to dislodge the fecal material completely from latrine pans, leading to low usage rates and poor maintenance (breaking/removing water seals). The new latrines were, therefore, less convenient than previously owned unimproved pit latrines. Furthermore, household members reported that they continued to use their existing latrines out of habit, kept them for visitors, outsiders and neighboring non-recipient households, many lacking a functional water seal (Fig. 3c) despite the availability of trial-provided improved latrines.

The qualitative team worked with CHWs and the latrine installation team to address these issues. In home visits and courtyard meetings, they demonstrated how the siphon/water seal functioned, and stressed the need to pour additional water with force to flush the feces through the water seal. They also discussed the advantage of having a functional water seal, citing reduced bad odors, and fewer flies and mosquitoes coming from the pit while a person was using the toilet. To discourage continued use of previously existing "unhygienic" (lacking functioning water seal) latrines, they were structurally closed with the household's consent to prevent ongoing environmental contamination.

\section{Investigation of shortfall in sanitation implementation benchmarks: Unsafe child feces disposal}

Prior to potty distribution, $>90 \%$ children aged $<3$ years were habitually defecating in the courtyard (Mahfuza Islam, manuscript in preparation). Qualitative investigation

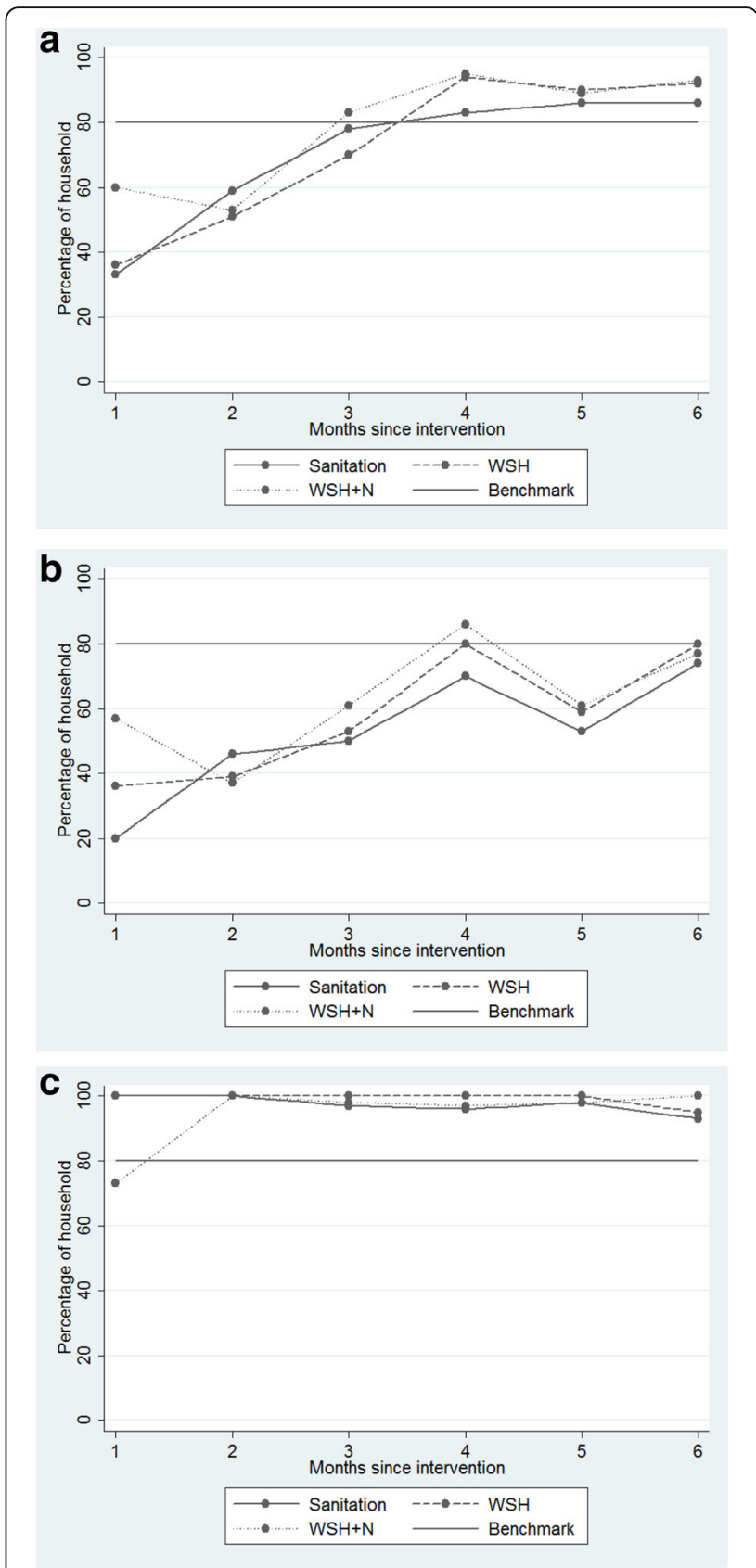

Fig. 3 Sanitation fidelity measures (\% households $n=176$ ) per month, for first to sixth monthly assessments, and a priori benchmark, WASH Benefits, Bangladesh 2012-2015. a households in which latrines were found with an intact water seal (both existing and icddr,b). b households in which pre-existing latrines were found with an intact water seal. c households in which trial-provided latrines were found with an intact water seal

revealed that although potties were provided and promoted according to protocol guidelines, additional support was required to encourage and teach rural mothers how to train their children to consistently use the potty. In addition, CHWs encouraged the mothers to store potties in a place that was accessible to the trained toddler when 
they need to use it; they encouraged household members other than the mother to participate in the potty training and habit formation. In addition, we provided refresher training to the CHWs to address safe feces disposal into latrines or covered pits, instead of the prevalent practices of disposing feces into the bushes just out of sight.

\section{Discussion}

We have summarized the implementation fidelity system put in place for the WASH Benefits Bangladesh efficacy trial, and provided an example of how systematic fidelity measures can detect compliance and deviations and trigger a rapid course correction. Such careful monitoring is especially essential in complex community-based efficacy studies where high uptake of technologies and behaviors is necessary to achieve the study aims [13]. Deviations in fidelity for large-scale interventions can also be detected through internal monitoring and evaluation, but this often suffers from slow report turnaround and insufficient review for critical feedback. An objective fidelity review with rapid assessment of initial rollout can be done in parallel to implementation process documentation and monitoring, to provide critical input and focus resources for increased efficiency.

Complex interventions tend to have lower fidelity because they are comparatively more difficult to implement than simpler interventions $[1,19]$. This is consistent with our findings for the sanitation intervention in the WASH Benefits Bangladesh study that included labor-intensive, multi-stepped latrine installation, close supervision, and quality assurance. The sanitation team installed 4533 latrines, for enrolled households and their neighbors in 21 sub-districts across four districts over 15 months. In our case, fidelity measurements demonstrated that the intervention was implemented as intended by the sixth assessment month; however, this required qualitative investigation and adjustments to the respective behavioral-change package.

Providing enabling products can encourage target behaviors; product quality influences uptake or response among intervention recipients [20]. In our case, households found the project-provided latrine of good quality but less convenient when supplied with a water seal, which some subsequently broke or removed. Breaking or removing water seals has been observed in Bangladesh [21] and India [22] and is believed to be common; however, there are few published data on the frequency of this practice. Determining how commonly this occurs could benefit behavior-change efforts in future latrine interventions in South Asia and among low-income countries where this practice may occur. Latrines with broken or missing water seals have a reduced ability to keep excreta separate from the environment and as such are categorized as unimproved [23].
We did not develop an implementation fidelity score or index to communicate the results in the monthly reports. A numerical index or score calculated for each assessment round might be useful in demonstrating the level of fidelity across combined intervention arms compared to individual intervention arms in a multi-factorial design as in WASH Benefits. However, the specific objective of early fidelity measures was to trigger investigation to address low uptake. A fidelity or implementation index may be relevant for explaining intervention uptake over the 24-month intervention period, to assist interpreting health impact.

In summary, fidelity indicators were efficient, quick to collect, easily observable and indicated the presence, functionality of hardware, condition and sign of use, and corresponding behaviors, which focused on technology accessibility, behavior-change communication crucial for sustained intervention uptake. Only the indicators for child feces disposal and CHW-delivered nutrition messages were reported and not directly observed and were, therefore, potentially overestimated. Fidelity assessment was conducted by only three research assistants who were not directly involved in intervention delivery thus data were generated at relatively low cost. Fidelity indicators described here can be used in other similar intervention trials.

\section{Conclusions}

An intensive implementation fidelity monitoring and response system proved beneficial for the WASH Benefits efficacy trial. Timely exploration of benchmark shortfalls enabled corrective measures to ensure that implementation adhered to the intended protocol. The indicators used were derived from easily observable, easy-to-collect data that can be used in other similar WASH and nutrition trials. Flexible adaptations to behavioral interventions at the community level can be directed by time-sensitive fidelity assessments and need to be documented to inform future large-scale studies in similar settings.

Although fidelity assessments are often conducted during the pilot or developmental stages of a study, the integrity of research studies of all designs and stages would benefit from a structured fidelity assessment as a key component $[19,24]$. To implement a routine program at scale requires further research into an adaptation of fidelity monitoring that supports program effectiveness.

\section{Abbreviations}

BCC: Behavior-change communication; CHW: Community health worker; VERC: Village education resource center; WSH: Water, sanitation, and hygiene

\section{Acknowledgements}

The authors acknowledge the WASH Benefits study collaborators and acknowledge the cooperation of the study households and communities that were visited several times for intervention delivery and data collection. 


\section{Funding}

This research study was funded by the Bill \& Melinda Gates Foundation grant OPPGD759 through the University of California Berkeley. icddr,b acknowledges with gratitude the commitment of the Bill \&Melinda Gates Foundation to its research efforts. Icddr,b is also grateful to the Governments of Bangladesh, Canada, Sweden and the UK for providing core/unrestricted support.

Availability of data and materials

Data can be made available through the authors.

\section{Authors' contributions}

MR drafted the manuscript with the listed co-authors. SPL, the principal investigator, drafted the research protocol with input from co-authors and some members of the Technical Advisory Group, and coordinated the study team. The intervention technology and behavior components, and the delivery methods were developed by PW, EL, FB, FH, SA, LU, TC, SMP, AMN, AKMM, and KKD with input from the Technical Advisory Group. All authors provided feedback, read and approved the manuscript.

\section{Ethics approval and consent to participate}

All households provided written informed consent at enrollment. The protocol was reviewed and approved by human subjects review committees at the International Centre for Diarrhoeal Disease Research, Bangladesh (icddr,b) and at the University of California, Berkeley.

\section{Consent for publication}

All co-authors have reviewed this version of the manuscript and provided consent for manuscript submission.

\section{Competing interests}

The authors declare that they have no competing interests.

\section{Publisher's Note}

Springer Nature remains neutral with regard to jurisdictional claims in published maps and institutional affiliations.

\section{Author details}

${ }^{1}$ Environmental Intervention Unit, Enteric and Respiratory Disease Program, Infectious Disease Division, International Centre for Diarrheal Disease Research, Bangladesh (icddr,b), Dhaka, Bangladesh. ${ }^{2}$ Johns Hopkins Bloomberg School of Public Health, Baltimore, MD, USA. ${ }^{3}$ Rollins School of Public Health, Emory University, Atlanta, GA, USA. ${ }^{4}$ Division of Infectious Diseases and Geographic Medicine, Stanford University, Stanford, CA, USA.

\section{Received: 11 April 2017 Accepted: 25 May 2018}

\section{Published online: 06 July 2018}

\section{References}

1. Greenhalgh T, Robert G, Macfarlane F, Bate P, Kyriakidou O. Diffusion of innovations in service organizations: systematic review and recommendations. Milbank Q. 2004;82:581-629.

2. Hohmann AA, Shear MK. Community-based intervention research: coping with the "noise" of real life in study design. Am J Psychiatry. 2002;159:201-7.

3. Proctor E, Silmere H, Raghavan R, Hovmand P, Aarons G, Bunger A, Griffey R, Hensley M. Outcomes for implementation research: conceptual distinctions, measurement challenges, and research agenda. Adm Policy Ment Health Ment Health Serv Res. 2011;38:65-76.

4. Carroll C, Patterson M, Wood S, Booth A, Rick J, Balain S. A conceptual framework for implementation fidelity. Implement Sci. 2007;2:1-9.

5. Dusenbury L, Brannigan $R$, Falco $M$, Hansen WB. A review of research on fidelity of implementation: implications for drug abuse prevention in school settings. Health Educ Res. 2003;18:237-56.

6. Breitenstein SM, Fogg L, Garvey C, Hill C, Resnick B, Gross D. Measuring implementation fidelity in a community-based parenting intervention. Nurs Res. 2010;59:158.

7. Cross W, West J. Examining implementer fidelity: conceptualising and measuring adherence and competence. J Children's Serv. 2011;6:18-33.

8. Durlak JA, DuPre EP. Implementation matters: a review of research on the influence of implementation on program outcomes and the factors affecting implementation. Am J Community Psychol. 2008:41:327-50.
9. Hawe P, Shiell A, Riley T, Gold L. Methods for exploring implementation variation and local context within a cluster randomised community intervention trial. J Epidemiol Community Health. 2004;58:788-93.

10. Rychetnik L, Frommer M, Hawe P, Shiell A. Criteria for evaluating evidence on public health interventions. J Epidemiol Community Health. 2002;56:119-27.

11. Mihalic S, Fertman Cl, Snyder SM, Jensen PS. Emotional \& Behavioral Disorders in Youth. 2004

12. Arnold BF, Null C, Luby SP, Unicomb L, Stewart CP, Dewey KG, Ahmed T, Ashraf S, Christensen G, Clasen T, et al. Cluster-randomised controlled trials of individual and combined water, sanitation, hygiene and nutritional interventions in rural Bangladesh and Kenya: the WASH benefits study design and rationale. BMJ Open. 2013;3:e003476.

13. Unicomb L, Begum F, Leontsini E, Rahman M, Ashraf S, Naser AM, Nizama F, Kannat K, Hussain F, Parvez SM, et al. WASH Benefits Study Bangladesh; management structure for achieving high coverage in an efficacy trial. 2018 (accepted)

14. P arvez SM, Azad R, Rahman R, Unicomb L, Ram PK, Naser AM, Stewart C, Jannat K, Rahman MJ, Leontsini E, et al. Achieving optimal technology and behavioral uptake of single and combined interventions in an efficacy trial (WASH Benefits) in rural Bangladesh. 2018. (accepted)

15. Hussain F, Clasen T, Akter S, Bawel V, Luby SP, Leontsini E, Unicomb L, Kanti BM, Brittany T, Winch PJ. Advantages and limitations for users of double pit pour-flush latrines in rural Bangladesh. BMC Public Health. 2017;17(1):515.

16. Hussain F, Luby SP, Unicomb L, Leontsini E, Naushin T, Buckland AJ, Winch PJ. Assessment of the acceptability and feasibility of child potties for safe child feces disposal in rural Bangladesh. Am J Trop Med Hygiene. 2017; 97(2):469-76.

17. Sultana R, Mondal UK, Rimi NA, Unicomb L, Winch PJ, Nahar N, Luby SP. An improved tool for household faeces management in rural Bangladeshi communities. Tropical Med Int Health. 2013;18:854-60.

18. Hulland KR, Leontsini E, Dreibelbis R, Unicomb L, Afroz A, Dutta NC, Nizame FA, Luby SP, Ram PK, Winch PJ. Designing a handwashing station for infrastructure-restricted communities in Bangladesh using the integrated behavioural model for water, sanitation and hygiene interventions (IBMWASH). BMC Public Health. 2013;13:877

19. Gearing RE, El-Bassel N, Ghesquiere A, Baldwin S, Gillies J, Ngeow E. Major ingredients of fidelity: a review and scientific guide to improving quality of intervention research implementation. Clin Psychol Rev. 2011;31:79-88.

20. Dreibelbis R, Winch PJ, Leontsini E, Hulland KR, Ram PK, Unicomb L, Luby SP. The integrated behavioural model for water, sanitation, and hygiene: a systematic review of behavioural models and a framework for designing and evaluating behaviour change interventions in infrastructure-restricted settings. BMC Public Health. 2013;13:1015.

21. Akter T, Ali AR, Dey NC. Transition overtime in household latrine use in rural Bangladesh: a longitudinal cohort study. BMC Public Health. 2014;14:721.

22. Routray P, Schmidt WP, Boisson S, Clasen T, Jenkins MW. Socio-cultural and behavioural factors constraining latrine adoption in rural coastal Odisha: an exploratory qualitative study. BMC Public Health. 2015;15:880.

23. World Health Organization, \& UNICEF. Progress on drinking water, sanitation and hygiene: 2017 update and SDG baselines. World Health Organization; 2017. http://www.who.int/mediacentre/news/releases/2017/launch-versionreport-jmp-water-sanitationhygiene.pdf. Accessed 19 June 2018.

24. Rounsaville BJ, Carroll KM, Onken LS. A stage model of behavioral therapies research: getting started and moving on from stage I. Clin Psychol Sci Pract. $2001 ; 8: 133-42$.

\section{Ready to submit your research? Choose BMC and benefit from}

- fast, convenient online submission

- thorough peer review by experienced researchers in your field

- rapid publication on acceptance

- support for research data, including large and complex data types

- gold Open Access which fosters wider collaboration and increased citations

- maximum visibility for your research: over $100 \mathrm{M}$ website views per year

\section{At BMC, research is always in progress.}

Learn more biomedcentral.com/submissions 